\title{
Predicting recurrence and metastasis of primary esophageal cancer with or without lymph node metastasis
}

\author{
TAKAYUKI TAJIMA $^{1}$, MASAYA MUKAI ${ }^{2}$, SHINKICHI SATO $^{3}$, HIROMI NINOMIYA ${ }^{3}$, KANAKO WAKUI $^{3}$, \\ NOBUKAZU KOMATSU ${ }^{3}$, KAZUTOSHI TSUCHIYA ${ }^{4}$, HISAO NAKASAKI ${ }^{5}$ and HIROYASU MAKUUCHI ${ }^{1}$ \\ ${ }^{1}$ Department of Surgery, Tokai University School of Medicine, Bohseidai, Isehara, Kanagawa 259-1193; \\ ${ }^{2}$ Department of Surgery, Tokai University Hachioji Hospital, 1838 Ishikawa-cho, Hachioji, Tokyo 192-0032; \\ Departments of ${ }^{3}$ Pathology, ${ }^{4}$ Endoscopy Center and ${ }^{5}$ Surgery, Tokai University Oiso Hospital, \\ 21-1 Gakyou, Oiso, Kanagawa 259-0198, Japan
}

Received October 18, 2005; Accepted December 23, 2005

\begin{abstract}
We studied 42 patients, consisting of 11 stage I/II patients who were node-negative $\left(\mathrm{LN}^{-}\right)$and 31 stage II/III patients who were node-positive $\left(\mathrm{LN}^{+}\right)$. In the $\mathrm{LN}^{-}$patients, detection of occult neoplastic cells (ONCs) in lymph nodes had a sensitivity of $0.0 \%(0 / 5)$, a false-positive (FP) rate of $33.3 \%(2 / 6)$, a specificity of $66.7 \%(4 / 6)$, a false-negative $(\mathrm{FN})$ rate of $100 \%(5 / 5)$, a positive predictive value (PPV) of $0.0 \%(0 / 2)$, and a negative predictive value (NPV) of $44.4 \%$ (4/9). In the $\mathrm{LN}^{+}$patients, the sensitivity of ONCs was $25.0 \%$ $(5 / 20)$, FP rate was $36.4 \%$ (4/11), specificity was $63.6 \%$ (7/11), FN rate was $75.0 \%(15 / 20)$, PPV was $55.6 \%(5 / 9)$, and NPV was $31.8 \%(7 / 22)$. In $\mathrm{LN}^{-}$patients, positivity for at least 2 of the 3 high-risk criteria had a sensitivity of $20.0 \%$ $(1 / 5)$, FP rate of $16.7 \%(1 / 6)$, specificity of $83.3 \%(5 / 6)$, FN rate of $80.0 \%(4 / 5), \mathrm{PPV}$ of $50.0 \%(1 / 2)$, and NPV of $55.6 \%$ (5/9). In $\mathrm{LN}^{+}$patients, these criteria had a sensitivity of $75.0 \%(15 / 20)$, FP rate of $9.1 \%(1 / 11)$, specificity of $90.9 \%$ $(10 / 11), \mathrm{FN}$ rate of $25.0 \%(5 / 20)$, PPV of $93.8 \%(15 / 16)$, and NPV of $66.7 \%(10 / 15)$. These results suggest that the high-risk criteria may be useful for predicting recurrence or metastasis in stage II/III lymph node-positive patients with esophageal cancer.
\end{abstract}

Correspondence to: Dr Takayuki Tajima, Department of Surgery, Tokai University Hospital, Bohseidai, Isehara, Kanagawa 259-1193, Japan

E-mail: takayukitajima1970@yahoo.co.jp

Abbreviations: LN, lymph node; ONCs, occult neoplastic cells; FP, false-positive; FN, false-negative; PPV, positive predictive value; NPV, negative predictive value

Key words: esophageal cancer, lymph node metastasis, occult neoplastic cells, isolated tumor cells, micrometastasis, high-risk group, occult systemic metastasis

\section{Introduction}

Lymph node (LN) metastasis is known to be the most important clinical indicator of the prognosis in patients with primary squamous esophageal cancer as well as patients with cancer of the breast, lung, stomach, and colon/rectum (1-4). In particular, LN metastasis of breast cancer has been investigated in detail, making it possible to clearly distinguish local from systemic disease and to decide on appropriate treatment methods and chemotherapy regimens by determining the presence or absence of LN metastasis $(5,6)$. Previous studies have shown that about $20-25 \%$ of patients who have primary esophageal cancer without LN metastasis classified as stage I or II and undergo curative resection develop life-threatening recurrence/metastasis that affects the liver or lungs postoperatively (7-9). Moreover, other studies have shown that about $40-50 \%$ of esophageal cancer patients with LN metastasis classified as stage II or III who undergo curative resection develop fatal hematogenous metastasis/recurrence in distant organs postoperatively $(7,10)$. It has been suggested that fatal hematogenous metastasis to the liver or lungs may develop after curative resection of esophageal cancer when tumor cells that are disseminated throughout the body during the perioperative period survive and grow in these distant organs $(3,4)$. Many studies have shown that the presence of cytokeratinpositive occult neoplastic cells (ONCs) floating in the sinuses of LNs far from the primary tumor is closely related to distant metastasis/recurrence (11-17). ONCs are very small numbers of malignant cells that cannot be detected in body fluids such as the portal blood or peripheral blood without complex techniques, but are trapped in the microcirculation of lymph nodes surrounding the primary tumor and can be semiquantitatively counted by using a relatively easy immunostaining technique (1-4).

To detect patients without LN metastasis who have an increased risk of the recurrence/metastasis of stage I/II cancer of the breast, stomach, or colorectal region, it has been reported that those who fulfill at least 2 of the following 3 criteria can be classified as a high-risk group: i) positivity for both lymphatic invasion (ly) and venous invasion (v) in the primary tumor; ii) dissection of $\leq 14$ lymph nodes at operation; and 


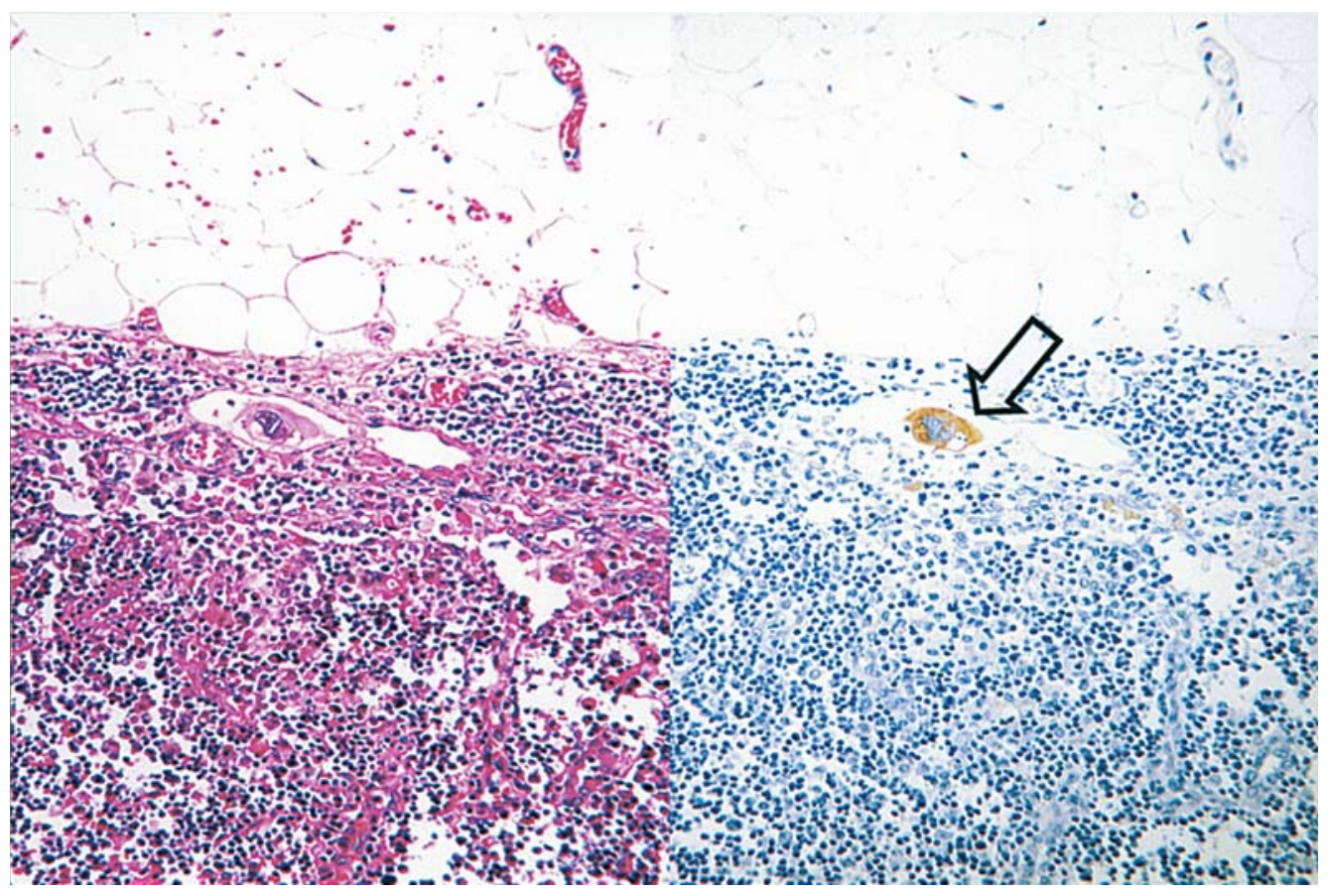

Figure 1. Cytokeratin-positive occult neoplastic cells freely floating in the peripheral lymph node sinuses of a patient from the recurrence group with pT2n1, stage II node-positive esophageal cancer (left, H\&E stain, x200; right, AE1/AE3 stain, x200).

iii) positivity for ONCs $(11,14,15,17)$. On the other hand, to identify a high-risk group for the recurrence/metastasis of stage II/III cancer of the breast, stomach, or colorectal region with LN metastasis, it seems appropriate to include patients who meet at least 2 of the following 3 criteria: i) exclusion of patients with D2 or D3 (D means the dissection number, which identifies the extent of LN dissection) and >n1 (n, number, which indicates the extent of LN metastasis) who have fewer than 3 metastatic nodes ( $<3$ nodes); ii) inclusion of patients with serosal exposure ( $>$ se) or adventitial exposure $(>\mathrm{a} 2)$, or with a tumor diameter $>2 \mathrm{~cm}(>\mathrm{t} 2)$; and iii) inclusion of those with ONCs in the LN sinuses $(12,13,16)$.

However, no study has examined the relationship between recurrence/metastasis of stage I/II node-negative or stage II/III node-positive esophageal cancer and the above-mentioned high-risk criteria including ONCs. Therefore, this study was designed to examine the prediction of distant metastasis or recurrence of primary esophageal cancer in patients with or without LN metastasis using ONCs or the high-risk criteria for recurrence/metastasis of gastric and colorectal cancer.

\section{Materials and methods}

Among patients who underwent curative resection of primary esophageal cancer from 1987 to 1999 , there were a total of 42 patients: i) in whom recurrence and survival could be determined from complete and reliable medical records and ii) who had at least 10 histologically negative lymph nodes without metastasis on routine H\&E staining. They consisted of 11 stage I/II node-negative patients and 31 stage II/III node-positive patients. They were also divided into 25 patients ( $\mathrm{LN}^{-}$in 5 cases, $\mathrm{LN}^{+}$in 20 cases) who developed recurrence/ metastasis of cancer within 5 years after surgery (recurrence group) and 17 patients ( $\mathrm{LN}^{-}$in 6 cases, $\mathrm{LN}^{+}$in 11 cases) who survived for at least 5 years postoperatively without recurrence (non-recurrence group).

Detection of ONCs. In the recurrence and non-recurrence groups, detection of ONCs was examined to calculate the sensitivity (true-positive rate), specificity (true-negative rate), false-positive (FP) rate, false-negative (FN) rate, accuracy, positive predictive value (PPV), and negative predictive value (NPV) for metastasis/recurrence. Cytokeratin immunostaining of harvested LNs was performed by a routine indirect immunoperoxidase method using a monoclonal anti-cytokeratin antibody (AE1/AE3; Fuji Chemical Industries, Ltd., Japan) $(18,19)$. A total of 10 nodes that were negative for metastasis by routine $\mathrm{H} \& \mathrm{E}$ staining were randomly selected from the dissected specimens of each patient, so 420 nodes were selected for the 42 patients. Serial sections of each LN specimen were stained with H\&E and keratin-AE1/AE3 to determine the positive rate and positive cell count for each stain. Tumor cells and/or tumor cell nests that were attached to or growing in the nodes were completely excluded, and only tumor cells floating freely in the LN sinuses were counted as ONCs (Fig. 1). After immunostaining for cytokeratin, the presence of 3 or more cytokeratin-positive cells was defined as a positive result, while the presence of 0-2 cytokeratin-positive cells was defined as negative (1-4).

Stratification into high-risk and low-risk groups for stage I/II node-negative esophageal cancer. Patients who met at least 2 of the following 3 criteria were assigned to the high-risk group for recurrence/metastasis: i) both lymphatic invasion (ly) and venous invasion (v) were positive in the primary tumor, ii) $\leq 14$ lymph nodes were dissected at surgery, and iii) ONCs were detected in the LN sinuses by cytokeratin immunostaining (Table I). The sensitivity, specificity, FP rate, FN rate, 
Table I. Criteria for separating patients into high-risk and lowrisk groups for the recurrence/metastasis of stage I/II nodenegative $\left(\mathrm{LN}^{-}\right)$esophageal cancer.

\begin{tabular}{lll}
\hline & \multicolumn{2}{c}{ Recurrence and metastasis } \\
\cline { 2 - 3 }$(\geq 2$ factors $)$ & \multicolumn{1}{c}{ Low-risk group } & High-risk group \\
\hline \multirow{3}{*}{ LN(-) } & Others & $\begin{array}{l}\text { ly }(+) \text { and } \mathrm{v}(+) \\
\leq 14 \text { nodes } \\
\end{array}$ \\
& LN dissection $\geq 15$ nodes & $\begin{array}{l}\text { Occult neoplastic cells }(-) \\
(+)\end{array}$ \\
\hline
\end{tabular}

LN, lymph node; ly, lymphatic invasion; v, venous invasion.

Table II. Criteria for separating patients into high-risk and lowrisk groups for the recurrence/metastasis of stage II/III nodepositive $\left(\mathrm{LN}^{+}\right)$esophageal cancer.

\begin{tabular}{lll}
\hline & \multicolumn{2}{c}{ Recurrence and metastasis } \\
\cline { 2 - 3 }$(\geq 2$ factors $)$ & \multicolumn{1}{c}{ Low-risk group } & High-risk group \\
\hline \multirow{3}{*}{$\mathrm{LN}(+)$} & $\mathrm{D} 2,3>\mathrm{n} 1(<3$ nodes $)$ & Others \\
& Tumor invasion $\leq \mathrm{pT} 2$ & $\geq \mathrm{pT} 3$ \\
& Occult neoplastic cells $(-)$ & $(+)$ \\
\hline
\end{tabular}

$\mathrm{n}$, histological extent of lymph node (LN) metastasis; pT2, proper muscle invasion; pT3, adventitial invasion.

accuracy, PPV, and NPV of these criteria were calculated and compared with the results obtained for prediction of recurrence using ONCs $(11,14,15,17)$.

Stage II/III node-positive esophageal cancer. Patients who met at least 2 of the following 3 criteria were assigned to a high-risk group for recurrence/metastasis: i) patients with D2 or D3 and >n1 ( $<3$ metastatic nodes) were excluded; ii) the histological depth of tumor invasion was not subadventitial $(<\mathrm{pT} 2)$, but was adventitial or more advanced ( $>$ pT3); and iii) ONCs were detected in the LN sinuses by cytokeratin immunostaining (Table II). The sensitivity, FP rate, specificity, FN rate, accuracy, PPV, and NPV of these criteria were calculated and compared with those for prediction of recurrence using ONCs $(12,13,16)$. The same parameters were also calculated for ONC-positive patients who fitted either of the remaining 2 criteria.

\section{Results}

ONCs for stage I/II node-negative esophageal cancer. None of the patients in the recurrence group were positive for ONCs, indicating a sensitivity of $0.0 \%(0 / 5)$ and an $\mathrm{FN}$ rate of $100.0 \%$ $(5 / 5)$ (Table III). In the non-recurrence group, 2 out of 6 patients were positive for ONCs and the remaining 4 patients were negative, so the FP rate was $33.3 \%(2 / 6)$ and the specificity was $66.7 \%(4 / 6)$. In addition, the accuracy was $36.4 \%$, while the PPV and NPV were $0.0 \%(0 / 2)$ and $44.4 \%$ (4/9), respectively (Table III).

Stage II/III node-positive esophageal cancer. In the recurrence group, 5 out of 20 patients were positive for ONCs and the remaining 15 patients were negative, indicating a sensitivity of $25.0 \%(5 / 20)$ and an FN rate of $75.0 \%(15 / 20)$ (Table IV). In the non-recurrence group, 4 out of 11 patients were positive for $\mathrm{ONCs}$ and the remaining 7 patients were negative, indicating an FP rate of $36.4 \%$ (4/11) and a specificity of $63.6 \%$ (7/11). The accuracy was $38.7 \%$, while the PPV and NPV were $55.6 \%$ (5/9) and $31.8 \%$ (7/22), respectively (Table IV).

High-risk criteria for stage I/II node-negative esophageal cancer. Regarding the high-risk criteria, 1 of the 5 patients in the recurrence group fulfilled at least 2 of the 3 criteria and the remaining 4 patients did not, indicating a sensitivity of $20.0 \%(1 / 5)$ and an FN rate of $80.0 \%$ (4/5) (Table III). In the non-recurrence group, 1 out of 6 patients fitted at least 2 of the 3 criteria and the remaining 5 patients did not, indicating an FP rate of $16.7 \%(1 / 6)$ and a specificity of $83.3 \%(5 / 6)$. The accuracy was $54.5 \%$, while the PPV and NPV were $50.0 \%$ $(1 / 2)$ and $55.6 \%(5 / 9)$, respectively (Table III).

Stage II/III node-positive esophageal cancer. In the recurrence group, 15 of the 20 patients met at least 2 of the 3 high-risk criteria and the remaining 5 patients did not, indicating a

Table III. Comparison of the sensitivity, specificity, accuracy, positive predictive value (PPV), and negative predictive value (NPV) for tumor recurrence/metastasis between detection of occult neoplastic cells (ONCs) and the high-risk criteria in patients who had stage I/II esophageal cancer without lymph node metastasis.

\begin{tabular}{|c|c|c|c|c|}
\hline \multirow[b]{2}{*}{ Total: $n=11$} & \multicolumn{2}{|c|}{$\%$ Occult neoplastic cells } & \multicolumn{2}{|c|}{$\%$ High-risk criteria } \\
\hline & $(+)$ & vs. & $(+)$ & vs. \\
\hline LN(-) cases; accuracy & 36.4 & & 54.5 & \\
\hline Recurrence $(n=5)$ & $0.0(0 / 5)$ & $100.0(5 / 5)$ & $20.0(1 / 5)$ & $80.0(4 / 5)$ \\
\hline \multirow[t]{2}{*}{ Non-recurrence $(n=6)$} & $33.3(2 / 6)$ & $66.7(4 / 6)$ & $16.7(1 / 6)$ & $83.3(5 / 6)$ \\
\hline & 0.0 (PPV) & 44.4 (NPV) & $50.0(\mathrm{PPV})$ & $55.6(\mathrm{NPV})$ \\
\hline
\end{tabular}

LN, lymph node; PPV, positive predictive value; NPV, negative predictive value. 
Table IV. Comparison of the sensitivity, specificity, accuracy, positive predictive value (PPV), and negative predictive value (NPV) for tumor recurrence/metastasis between detection of occult neoplastic cells (ONCs) and the high-risk criteria in patients who had stage II/III esophageal cancer with lymph node metastasis.

$\%$ Occult neoplastic cells

Total: $\mathrm{n}=31$

vs.

$(-)$
$\%$ High-risk criteria

(+) $\quad$ vs. (-)

\begin{tabular}{|c|c|c|c|c|}
\hline $\mathrm{LN}(+)$ cases; accuracy & 38.7 & & 80.6 & \\
\hline Recurrence $(n=20)$ & $25.0(5 / 20)$ & $75.0(15 / 20)$ & $75.0(15 / 20)$ & $25.0 \quad(5 / 20)$ \\
\hline \multirow[t]{2}{*}{ Non-recurrence $(n=11)$} & $36.4(4 / 11)$ & $63.6 \quad(7 / 11)$ & $9.1 \quad(1 / 11)$ & $90.9(10 / 11)$ \\
\hline & 55.6 (PPV) & 31.8 (NPV) & 93.8 (PPV) & 66.7 (NPV) \\
\hline
\end{tabular}

LN, lymph node; PPV, positive predictive value; NPV, negative predictive value.

Table V. The sensitivity, specificity, accuracy, positive predictive value (PPV), and negative predictive value (NPV) of the high-risk criteria for recurrence/metastasis in patients who were positive for all 3 criteria.

$\%$ Positive cases of occult neoplastic cells

Total: $\mathrm{n}=11$ 3 -factors $(+) \quad$ vs. others

\begin{tabular}{llll}
\hline Accuracy & 54.5 & & \\
Recurrence $(\mathrm{n}=5)$ & $20.0(1 / 5)$ & $80.0(4 / 5)$ & $50.0(1 / 2, \mathrm{PPV})$ \\
Non-recurrence $(\mathrm{n}=6)$ & $16.7(1 / 6)$ & $83.3(5 / 6)$ & $55.6(5 / 9, \mathrm{NPV})$
\end{tabular}

PPV, positive predictive value; NPV, negative predictive value.

sensitivity of $75.0 \%(15 / 20)$ and an FN rate of $25.0 \%(5 / 20)$ (Table IV). In the non-recurrence group, one of the 11 patients fulfilled at least 2 of the 3 criteria and the remaining 10 patients did not, indicating an FP rate of $9.1 \%(1 / 11)$ and a specificity of $90.9 \%(10 / 11)$. The accuracy was $80.6 \%$, while the PPV and NPV were $93.8 \%(15 / 16)$ and $66.7 \%(10 / 15)$, respectively (Table IV).

One of the 5 patients in the recurrence group was positive for ONCs and also fulfilled the other 2 criteria, but the remaining 4 patients did not meet all 3 criteria, indicating a sensitivity of $20.0 \%(1 / 5)$ and an FN rate of $80.0 \%(4 / 5)$ (Table V). Similarly, 1 of the 6 patients in the non-recurrence group was positive for ONCs and met the other 2 criteria, but the remaining 4 patients did not fulfill all 3 criteria, indicating an FP rate of $16.7 \%(1 / 6)$ and a specificity of $83.3 \%(5 / 6)$. The accuracy was $54.5 \%$, while the PPV and NPV were $50.0 \%$ $(1 / 2)$ and $55.6 \%$ (5/9), respectively (Table V).

\section{Discussion}

Stage I esophageal cancer without LN metastasis is virtually limited to the primary tumor. However, it is certain that, as in the case of gastric cancer and large bowel cancer, some viable cancer cells including circulating ONCs escape from the primary tumor and are disseminated throughout the body $(11,15,17)$. These malignant cells are generally destroyed after invading peripheral lymphatics or vessels because they are recognized as foreign and phagocytized by host immune cells (20-22). However, a very small number of cancer cells that escape from the host defenses may reach distant organs such as the liver or lungs and survive there, although the detailed mechanisms involved remain unclear. One of the mechanisms leading to systemic dissemination of cancer cells is vascular invasion in the primary tumor, which was included in our high-risk criteria. Positivity for ly and/or v clearly indicates infiltration of the primary tumor into the systemic circulation, but does not serve as a direct indicator of generalized tumor cell dissemination because ly and/or v are determined by microscopy of tumor sections and do not directly reflect the movements of cancer cells. In fact, most tumor cells that enter the circulation become trapped and are eliminated when the draining vessels are completely blocked by the primary cancer. If these cells survive, they are more likely to be involved in local infiltration of a nearby organ that enlarges the primary tumor rather than causing distant metastasis. ONCs are cytokeratin-positive tumor cells floating in lymph nodes distant from the primary tumor and these cells are considered to be a more reliable indicator of the systemic spread of cancer than ly and/or v positivity of the primary tumor.

Patients fear advanced cancer because it may recur to threaten their survival after surgical resection. Accordingly, the ability to predict that a particular tumor is unlikely to recur could greatly reduce the mental burden on patients. Earlier 
studies have shown that Japanese patients who undergo curative resection of primary esophageal cancer without LN metastasis have a 5-year survival rate of about $75-80 \%$, and that about $20-25 \%$ of them develop fatal distant metastasis in the liver or lungs (7-9). Since the above studies indicated a recurrence rate of about $25 \%$, it can be considered that esophageal cancer is unlikely to recur when it is surgically removed before lymph node metastasis has occurred. The highrisk criteria tested in the present study showed a specificity of $83.3 \%(5 / 6)$ for predicting the recurrence of node-negative esophageal cancer and $90.9 \%$ (10/11) for node-positive esophageal cancer. Therefore, these criteria may allow fairly reliable selection of patients with a low-risk of the recurrence of esophageal cancer, including node-positive patients. This could contribute to improving the quality of life by reducing the mental burden of most patients after surgery for esophageal cancer.

Esophageal cancer with lymph node metastasis has been reported to cause life-threatening distant metastasis/recurrence in around $40-50 \%$ of patients who undergo curative resection $(7,10)$. Therefore, accurately predicting the risk of recurrence/ metastasis and deciding the postoperative therapeutic strategy accordingly is quite important for high-risk patients. When the predictive value of ONCs for recurrence was tested in the present study, the sensitivity and PPV were only $25.0 \%(5 / 20)$ and $55.6 \%$ (5/9), respectively. Patients with esophageal cancer undergo surgical dissection of lymph nodes in the cervical, thoracic, and abdominal regions, with from 14 to 104 lymph nodes (the mean value; 48.6 nodes) being harvested. Although only 10 lymph nodes per patient were randomly selected in this study, the anatomical characteristics of the complicated esophageal lymphatic system suggest that at least 30 lymph nodes covering the three dissection fields should have been examined. In contrast, the high-risk criteria showed a sensitivity, PPV, and accuracy of $75.0 \%(15 / 20), 93.8 \%$ $(15 / 16)$, and $80.6 \%(25 / 31)$, respectively, for the detection of recurrence. Therefore, the high-risk criteria may provide a more accurate indication of the patients who are at risk of developing recurrence and thus help clinicians to decide about the early initiation of chemoradiotherapy, as well as the followup schedule for laboratory tests and imaging studies such as ultrasound and computed tomography.

Previous studies that examined the relationship between the number of metastatic lymph nodes and the prognosis have shown that the survival rate is lower in patients with 3 or more positive nodes $(3,20)$. This is probably because up to 2 metastatic nodes can be reliably removed along with the primary tumor by en bloc surgical dissection. In the present study, $81.8 \%(9 / 11)$ of the non-recurrence group of nodepositive patients had 1 or 2 metastatic nodes, while $75.0 \%$ $(15 / 20)$ of the recurrence group had 3 or more metastatic nodes. This finding suggests that $\mathrm{LN}$ metastasis is not always an indicator of systemic disease. The number of circulating tumor cells may be too small to cause recurrence/metastasis when there are up to 2 metastatic nodes. When the number of metastatic nodes increases to 3 or more, circulating tumor cells like ONCs may exist in sufficient numbers to proliferate at a distant site $(3,20)$. The concept of ONCs differs from that of micrometastasis, for which there is no consensus about the relationship with survival, possibly because of its unclear definition. In contrast, it has been reported that circulating ONCs should be considered as an aggressive form of occult systemic metastasis (23). Improvement of patient survival after recurrence is very important for increasing the overall survival rate of cancer and achieving a real survival benefit $(24,25)$. Since esophageal cancer is generally more aggressive than gastric/large bowel cancer, it should be more beneficial for patients undergoing curative resection of this cancer who have $\mathrm{LN}$ metastasis to receive accurate prediction of the risk of recurrence and be treated with systemic consolidation chemotherapy and/or chemoradiotherapy during the period when systemic metastasis is still occult before clinical recurrence develops (26-28). However, it will be necessary to study more patients in the future in order to better analyze the relationship between ONCs and survival, including 5-year relapse-free survival and overall survival.

\section{Acknowledgements}

This study was supported by grants from the Occult Neoplastic Cells Research and Study Group (\#2004-2291; Tokai University Hachioji Hospital, Hachioji, Tokyo, Japan) and the Research and Study Program of Tokai University Educational System General Research Organization (\#2005-48; Tokai University Hospital, Isehara, Kanagawa, Japan).

\section{References}

1. Mukai M, Sato S, Komatsu N, Nishida T, Shiba K, Ito I, Nakasaki $\mathrm{H}$ and Makuuchi $\mathrm{H}$ : Correlation between occult neoplastic cells in the lymph node sinuses and recurrence in patients with Dukes' C colorectal cancer. Oncol Rep 10: 1165-1169, 2003.

2. Mukai M, Sato S, Komatsu N, Nishida T, Shiba K, Ito I, Nakasaki $\mathrm{H}$ and Makuuchi $\mathrm{H}$ : Correlation between occult neoplastic cells in the lymph node sinuses and recurrence in patients with curatively resected Dukes' B colorectal cancer. Oncol Rep 10: 1177-1181, 2003.

3. Mukai M, Sato S, Nishida T, Komatsu N, Shiba K, Nakasaki H and Makuuchi H: Selection criteria for high-risk and low-risk groups of recurrence and metastasis in patients with primary colorectal cancer. Oncol Rep 10: 1753-1758, 2003.

4. Mukai M, Sato S, Nakasaki H, Saito Y, Nishiumi N, Iwasaki M, Tokuda Y, Ogoshi K, Inoue H and Makuuchi H: Occult neoplastic cells in the lymph node sinuses and recurrence of primary breast, lung, esophageal and gastric cancer. Oncol Rep 11: 81-84, 2004

5. National Institutes of Health Consensus Development Panel. National Institutes of Health consensus development conference statement: adjuvant therapy for breast cancer, November 1-3, 2000. J Natl Cancer Inst 93: 979-989, 2001.

6. Goldhirsch A, Glick JH, Gelber RD, Coates AS and Senn H-J: Meeting highlights: international consensus panel on the treatment of primary breast cancer. J Clin Oncol 19: 3817-3827, 2001.

7. Comprehensive Registry of Esophageal Cancer in Japan (1998, 1999) and Long-term Results of Esophagectomy in Japan (19881997). 3rd edition. The Japanese Society for Esophageal Diseases, 2002

8. Vazquez-Sequeiros E, Wang L, Burgart L, Harmsen W, Zinsmeister A, Allen M, Jondal M and Wiersema M: Occult lymph node metastases as a predictor of tumor relapse in patients with node-negative esophageal carcinoma. Gastroenterology 122: 1815-1821, 2002.

9. Gotohda N, Nishimura M, Yoshida J, Nagai K and Tanaka N: The pattern of lymphatic metastases in superficial squamous cell carcinoma of the esophagus. Hepatogastroenterology 52: 105-107, 2005.

10. Dhar DK, Tachibana M, Kinukawa N, Riruke M, Kohno H, Little AG and Nagasue N: Prognostic significance of lymph node size in patients with squamous esophageal cancer. Ann Surg Oncol 9: 1010-1016, 2002. 
11. Mukai M, Sato S, Komatsu N, Kimura T, Ninomiya H, Nakasaki H, Ogoshi K and Makuuchi M: Accuracy of criteria for predicting the recurrence and metastasis of stage I and II gastric cancer without lymph node metastasis. Oncol Rep 12: 59-62, 2004.

12. Mukai M, Sato S, Komatsu N, Kimura T, Ninomiya H, Nakasaki H, Ogoshi K and Makuuchi H: Accuracy of criteria for predicting recurrence and metastasis in stage II and III gastric cancer with lymph node metastasis. Oncol Rep 12: 63-66, 2004.

13. Mukai M, Sato S, Komatsu N, Kimura T, Ninomiya H, Nakasaki H, Ogoshi K and Makuuchi H: Predicting the recurrence/metastasis of stage II and III breast cancer with lymph node metastasis. Oncol Rep 12: 303-306, 2004.

14. Mukai M, Sato S, Komatsu N, Kimura T, Ninomiya H, Nakasaki H, Ogoshi $\mathrm{K}$ and Makuuchi H: Predicting the recurrence/metastasis of stage I and II breast cancer without lymph node metastasis. Oncol Rep 12: 745-748, 2004.

15. Mukai M, Sato S, Tajima T, Kimura T, Komatsu N, Ninomiya H, Nakasaki H, Ogoshi K and Makuuchi H: Predicting recurrence and metastasis of stage II/Dukes' B colorectal cancer without lymph node metastasis. Oncol Rep 12: 1127-1130, 2004.

16. Mukai M, Sato S, Tajima T, Kimura T, Komatsu N, Ninomiya H, Nakasaki H, Ogoshi K and Makuuchi H: Predicting recurrence and metastasis of stage III/Dukes' C colorectal cancer with lymph node metastasis. Oncol Rep 12: 1301-1304, 2004.

17. Mukai M, Sato S, Tajima T, Kimura T, Komatsu N, Ninomiya H, Nakasaki H, Ogoshi K and Makuuchi $\mathrm{H}$ : Predicting recurrence and metastasis of Dukes' A primary colorectal cancer with or without proper muscle invasion. Oncol Rep 12: 1305-1308, 2004.

18. Nakane PK and Pierce GB: Enzyme-labeled antibodies: preparation and application for localization of antigens. J Histochem Cytochem 14: 929-931, 1966.

19. Nakane PK and Pierce GB: Enzyme-labeled antibody for the light and electron microscopic localization of tissue antigens. J Cell Biol 33: 307-318, 1967.

20. Mukai M, Ito I, Mukoyama S, Tajima T, Saito Y, Nakasaki H, Sato $\mathrm{S}$ and Makuuchi H: Improvement of 10 -year survival by Japanese radical lymph node dissection in patients with Dukes' B and C colorectal cancer: a 17-year retrospective study. Oncol Rep 10: 927-934, 2003.
21. Mukai M, Tajima T, Nakasaki H, Sato S, Ogoshi K and Makuuchi H: Efficacy of postoperative adjuvant oral immunochemotherapy in patients with Dukes' B colorectal cancer. Ann Cancer Res Therap 11: 201-214, 2003.

22. Mukai M, Tajima T, Nakasaki H, Sato S, Ogoshi K and Makuuchi H: Efficacy of postoperative adjuvant oral immunochemotherapy in patients with Dukes' C colorectal cancer. Ann Cancer Res Therap 11: 215-229, 2003.

23. Mukai M: Occult neoplastic cells and malignant micro-aggregates in lymph node sinuses: review and hypothesis. Oncol Rep 14: 173-175, 2005.

24. Feinstein AR, Sosin DM and Wells CK: The Will Rogers phenomenon: stage migration and new techniques as a source of misleading statistics for survival in cancer. N Engl J Med 312: 1604-1608, 1985.

25. Bunt AMG, Hermans J, Smit VTHBM, van de Velde CJH, Fleuren GL and Bruijn JA: Surgical/pathologic stage migration confounds comparisons of gastric cancer survival rates between Japan and Western countries. J Clin Oncol 13: 19-25, 1995.

26. Mukai M, Sato S, Ninomiya H, Wakui K, Komatsu N, Tsuchiya K, Nakasaki H and Makuuchi H: Recurrence and 5-FU sensitivity of stage III/Dukes' C colorectal cancer with occult neoplastic cells in lymph node sinuses. Oncol Rep 14: 1165-1169, 2005.

27. Mukai M, Sato S, Ninomiya H, Wakui K, Komatsu N, Tsuchiya K, Nakasaki $\mathrm{H}$ and Makuuchi $\mathrm{H}$ : Recurrence and 5-FU sensitivity of stage II/Dukes' B colorectal cancer with occult neoplastic cells in lymph node sinuses. Oncol Rep 14: 1171-1176, 2005.

28. Mukai M, Sato S, Ninomiya H, Wakui K, Komatsu N, Tsuchiya K, Tajima T, Nakasaki H and Makuuchi H: Recurrence and 5-FU sensitivity of stage II/III node-positive gastric cancer with occult neoplastic cells in lymph node sinuses. Oncol Rep 14: 1505-1510, 2005. 